\title{
Impacto de la cintura hipertensiva en el riesgo coronario y cumplimiento de la meta colesterol LDL en adultos de Trujillo
}

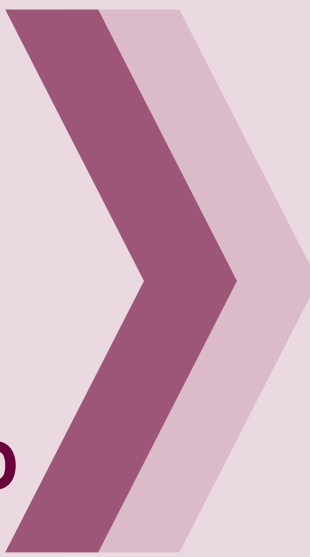

\author{
Impact of hypertensive waist in \\ coronary risk and compliance \\ with the LDL cholesterol target \\ in adults of Trujillo
}

Edgardo Linares-Reyes, ${ }^{1}$ Mayita Lizbeth Álvarez-Vargas, ${ }^{2}$

Karen Yanet Castillo-Minaya, ${ }^{3}$ Patricia Isabel Escobar-Pairazamán, ${ }^{4}$ Juan Jorge Huamán-Saavedra ${ }^{5}$

1 Médico. Centro de Atención Primaria Metropolitano, EsSalud, Trujillo, Perú.

2 Médica. Egresada de la Facultad de Medicina de la Universidad Nacional de Trujillo (UNT).

3 Médico. Magíster en Epidemiología. Residente de Patología Clínica, Hospital Nacional Arzobispo de Loayza, Lima, Perú.

4 Médico serumista, Centro de Salud de Chambamontera, Jaén, Cajamarca.

5 Médico patólogo clínico. Facultad de Medicina, UNT.

\section{RESUMEN}

INTRODUCCIÓN. La determinación de la cintura hipertensiva (CH) es propuesta como la primera etapa para el despistaje de síndrome metabólico y riesgo coronario. Oвjetivo. Determinar el impacto de la $\mathrm{CH}$ en el riesgo coronario y cumplimiento de la meta colesterol LDL en adultos de Trujillo, Perú. Pacientes y método. Estudio descriptivo, observacional, analítico, prospectivo realizado en 552 personas, 276 varones y 276 mujeres dividido en grupos etarios de 20-39, 40-59, 60-79 años. Se determinaron la presión arterial, cintura, glicemia y perfil lipidico. Se consideró CH si PAS $\geq 130 \mathrm{~mm} \mathrm{Hg}$, PAD $\geq 85 \mathrm{mmHg} 0$ antecedentes de hipertensión arterial, $y$ cintura $\geq 94 \mathrm{~cm}$ en varones $y \geq 80 \mathrm{~cm}$ en mujeres; las categorías de riesgo coronario y las metas de LDL según el Adult Treatment Panel III. Resultados. 126 personas con $\mathrm{CH}(23,14 \%), 55$ varones $(19,92 \%)$ y 71 mujeres $(26,44 \%)$; la categoría baja fue $27,27 \%$ en varones y $39,73 \%$ en mujeres; la alta, 30,91 y $15,07 \%$, respectivamente. $\operatorname{Sin} \mathrm{CH}$, la categoría de riesgo baja fue en varones $65,16 \%$ y en mujeres $84,65 \%$ y la alta 10,41 y $4,95 \%$ respectivamente. El cumplimiento de la meta de LDL fue: con $\mathrm{CH}$ en varones $56,36 \%$, mujeres $31,51 \%$; $\sin \mathrm{CH} 87,33$ y $72,77 \%$ respectivamente. Conclusiones. El impacto de la presencia de cintura hipertensiva es expresado al encontrar una mayor frecuencia de categoría de riesgo moderadamente alto y alto y un menor cumplimiento de la meta LDL en las categorías de riesgo bajo, moderado y moderadamente alto.

Palabras clave: hipertensión arterial; riesgo coronario; colesterol LDL; corazón

\begin{abstract}
InTRODUCTION. Hypertensive waist (HW) have been reported as the first step of screening for metabolic syndrome and coronary risk. Овлестіve. To determine the Impact of HW in coronary risk and compliance with the LDL cholesterol target in adults of Trujillo, Perú. Patients and method. Descriptive, observational, analytic and prospective study. 552 people, 276 men and 276 women divided in groups of 20-39, 40-59 and of 60-79 years old. Blood pressure, waist, blood glucose and lipid profile were determined. HW was considered if systolic $\mathrm{BP} \geq 130 \mathrm{~mm} \mathrm{Hg}, \mathrm{PAD} \geq 85 \mathrm{~mm} \mathrm{Hg}$ or hypertension history, waist $\geq 94 \mathrm{~cm}$ in men and $\geq 80 \mathrm{~cm}$ in women; the coronary risk level and LDL goal according to Adult Treatment Panel III. Results.. 126 HW cases (23.14\%), 55 in men (19.92\%) and 71 in women (26.44\%). In HW men the low level of risk coronary was $27.27 \%$ and $39.73 \%$ in women, however the high level were $30.91 \%$ and $15.07 \%$ respectively. In without $\mathrm{HW}$ men the low level of risk coronary was $65.16 \%$ and $84.65 \%$ in women, and the high level 10.41 and $4,95 \%$ respectively. The LDL cholesterol goals in HW men was achieved in $56.36 \%$ and $31.51 \%$ in women, and in people without HW were $87.33 \%$ and $72.77 \%$ respectively. Conclusions: The impact of the presence of hypertensive waist is expressed on having found a major frequency of category of risk moderately high and high and a minor compliance of the LDLC cholesterol goal in the categories of low, moderate and moderately high risk.
\end{abstract}

KEY WORDS: arterial hypertension; coronary risk; LDL cholesterol; heart 


\section{INTRODUCCIÓN}

La cintura hipertensiva es definida como una presión arterial sistólica $\geq 130 \mathrm{mmHg}$ o una presión arterial diastólica $\geq 85 \mathrm{mmHg} 0$ antecedentes de hipertensión arterial en tratamiento, más una circunferencia de la cintura $\geq 80 \mathrm{~cm}$ en mujeres y $\geq 94 \mathrm{~cm}$ para hombres, según el estudio realizado por Nita. ${ }^{1}$

El riesgo coronario se define como la probabilidad de presentar un evento coronario en los próximos diez años, según la tabla de Framingham. ${ }^{2}$ Existen cuatro categorías riesgo coronario: bajo, moderado, moderadamente alto y alto; ${ }^{2}$ en 2013 la ACC publicó una nueva directriz de prescripción de estatinas de acuerdo con las características clínicas y niveles de LDL. ${ }^{3}$

Las categorías de riesgo coronario son las siguientes: ${ }^{2,4}$ "alto riesgo" significa que más de $20 \%$ de individuos tendrán un evento recurrente de cardiopatía coronaria dentro de los próximos diez años; "riesgo moderadamente alto" significa que menos de $20 \%$ de individuos tendrán un evento de cardiopatía coronaria; "riesgo moderado" significa que menos de $10 \%$ de individuos tendrán un evento de cardiopatía coronaria y "riesgo bajo" significa que menos de $5 \%$ de individuos tendrán un evento de cardiopatía coronaria. ${ }^{5}$

Cada vez hay mayor evidencia que demuestra que existe riesgo coronario incluso desde niveles de presión arterial (PA) menores que las requeridas para diagnosticar hipertensión arterial (HTA).,6-8 El riesgo coronario se duplica con cada incremento de $20 \mathrm{mmHg}$ en la presión sistólica o $10 \mathrm{mmHg}$ en la presión diastólica.,8

El Programa Nacional de Educación en Colesterol (o NCEP) ha identificado al colesterol LDL como el blanco primario de tratamiento y también ha determinado la meta de colesterol LDL que se debe alcanzar, según la categoría de riesgo. ${ }^{4}$ Para cada categoría de riesgo existe un límite de LDL máximo permisible por encima del cual se debe administrar tratamiento, esta es la meta LDL. Posterior al ATP III, se realizaron varias investigaciones clínicas que mostraron la importancia de reducir el colesterol LDL, incluso hasta menos de $70 \mathrm{mg} /$ $\mathrm{dl}$, en pacientes con muy alto riesgo coronario. ${ }^{9-12}$
El "síndrome metabólico" (presencia de tres de cinco factores de riesgo que incluyen hiperglicemia basal, hipertensión arterial, obesidad central y dislipidemia aterogénica) ${ }^{4}$ también aumenta el riesgo coronario. ${ }^{13}$

En Trujillo, Huamán et al., ${ }^{14}$ en un estudio realizado en 246 adultos de 20 a 69 años, encontraron que el riesgo coronario fue bajo en $73,98 \%$; moderado, en el 5,28\%; moderadamente alto, en $11,79 \%$, y alto, en $8,94 \%$. La meta de colesterol LDL se logró alcanzar en $67,89 \%$ de los sujetos, independientemente del sexo. La edad, la frecuencia de hipertensión y diabetes y las concentraciones de colesterol fueron significativamente mayores en aquellos que no cumplieron la meta. En nuestro medio se han realizado diversos estudios para determinar la frecuencia de dislipidemia en la población adulta. También se logró identificar la incidencia de las diferentes categorías de riesgo coronario así como la tasa de sujetos que lograron alcanzar las metas de colesterol LDL recomendadas por ATP III. Al ser la cintura hipertensiva una expresión de dos aspectos esenciales del síndrome metabólico (hipertensión arterial e hipertrigliceridemia), ${ }_{1}^{1}$ y dado que no existen trabajos publicados que estudien la cintura hipertensiva como tal a nivel nacional, se plantea como objetivo principal determinar el impacto de la cintura hipertensiva en el riesgo coronario y cumplimiento de la meta colesterol LDL en adultos de Trujillo.

\section{PACIENTES Y MÉTODO}

Estudio epidemiológico de corte transversal, descriptivo, observacional, analítico. La población de estudio lo constituyeron las personas de raza mestiza con edades entre 20 y 79 años, residentes de la ciudad de Trujillo (distrito de Trujillo y las urbanizaciones aledañas), durante el período diciembre 2010-noviembre 2013. Los criterios de exclusión fueron gestación, diabetes tipo 1 y pacientes con alguna patología que altere la circunferencia de la cintura.

El tamaño de la muestra se obtuvo aplicando la fórmula para variable cualitativa para una población con $Z \quad / 2>1,96 ; 15 p=0,2$, de un estudio anterior, ${ }^{16}$ y con un margen de error de 0,05. La selección fue aleatoria simple hasta completar 276 personas de sexo masculino y 276 del femenino. 
La muestra fue distribuida equitativamente según sexo y edad; se formaron tres grupos etarios: de 20 a 39 años, de 40 a 59 años y de 60 a 79 años, cada uno con 92 personas.

- La edad es factor de riesgo coronario en varones si es $\geq 45$ años y en mujeres si $\geq 55$ años.

$\triangle$ El colesterol HDL es factor de riesgo coronario si es menor de $40 \mathrm{mg} / \mathrm{dl}$.

$\Delta$ El valor normal de los triglicéridos es $<150 \mathrm{mg} /$ $\mathrm{dl}$; existe hipertrigliceridemia si $\geq 150 \mathrm{mg} / \mathrm{dl}$.

$\Delta$ La glucosa sanguínea es expresada en $\mathrm{mg} / \mathrm{dl}$; la diabetes se considera por diagnóstico anterior 0 en tratamiento, o con glicemia basal $\geq 126 \mathrm{mg} /$ $\mathrm{dl}$, en dos oportunidades, siguiendo los criterios de la Asociación Americana de Diabetes.

- Tabaquismo: es riesgo el fumar cigarrillos desde uno por día.

$\triangle$ Presión arterial: se considera factor de riesgo si es hipertensión, con PA $\geq 140 / 90 \mathrm{mmHg} 0$ si el paciente está recibiendo tratamiento antihipertensivo.

- Antecedentes familiares de cardiopatía coronaria prematura: cardiopatía coronaria en un familiar de primer grado de sexo masculino antes de los 55 años, cardiopatía coronaria en un familiar de primer grado de sexo femenino antes de los 65 años.

$\Delta$ Riesgo a los 10 años: calculado según las tablas de Framingham.

\section{Categoría de riesgo coronario}

Nivel de riesgo coronario, que puede ser,

$\triangle$ De alto riesgo: cardiopatía coronaria o equivalente: diabetes, otras formas clínicas de enfermedad ateroesclerótica (arteriopatía periférica, aneurisma de la aorta abdominal y enfermedad sintomática de la arteria carótida), múltiples factores de riesgo que confieren un riesgo de cardiopatía coronaria a los 10 años $>20 \%$.

Incluye un subgrupo de muy alto riesgo: pacientes con cardiopatía coronaria más: 1) múltiples factores de riesgo mayores, especialmente diabetes; 2) factores de riesgo severos y mal controlados (especialmente tabaquismo); 3) factores de riesgo múltiples del síndrome metabólico (especialmente cuando los triglicéridos $\geq 200 \mathrm{mg} / \mathrm{dl}$, más colesterol no $\mathrm{HDL} \leq 130 \mathrm{mg} / \mathrm{dl}$, con HDL bajo y 4) síndrome coronario agudo.

$\Delta$ Riesgo moderadamente alto: dos o más factores de riesgo y riesgo a los diez años, de 10 a $20 \%$.

- Riesgo moderado: dos o más factores de riesgo y riesgo a los 10 años $<10 \%$.

- Riesgo bajo: uno o ningún factor de riesgo. Factores de riesgo (de 0 a 5): se consideran HDL, edad, tabaquismo, hipertensión, antecedentes familiares de cardiopatía coronaria.

Circunferencia de la cintura: medida en un punto medio entre las espinas iliacas y los rebordes costales inferiores después de una espiración completa.

Cintura hipertensiva: la presencia de una presión arterial sistólica $\geq 130 \mathrm{mmHg}$ o una presión arterial diastólica $\geq 85 \mathrm{mmHg} 0$ antecedentes de hipertensión arterial en tratamiento, más una circunferencia de la cintura $\geq 80 \mathrm{~cm}$ en mujeres y $\geq 94 \mathrm{~cm}$ para hombres.

Meta de colesterol LDL: para alto riesgo es < $100 \mathrm{mg} / \mathrm{dl}$ (muy alto < $70 \mathrm{mg} / \mathrm{dl}$ ); para riesgo moderadamente alto y moderado, $<130 \mathrm{mg} / \mathrm{dl}$ y riesgo bajo, < $160 \mathrm{mg} / \mathrm{dl} .1,4,14,17-23$

El impacto fue medido por la mayor frecuencia de las categorías alto riesgo y riesgo moderadamente alto en los pacientes con cintura hipertensiva, asimismo, por la menor frecuencia en el cumplimiento de la meta LDL.

\section{Procedimiento de recolección de datos}

Se llenó una ficha de datos (edad, sexo, cintura, presión arterial, antecedentes familiares y medicamentos). La circunferencia de la cintura fue medida en un punto medio entre las crestas iliacas y el reborde costal inferior después de realizar una espiración completa. La presión arterial fue medida con esfigmomanómetro aneroide, en el brazo no dominante y después de 10 minutos de reposo. Se tomaron tres lecturas y se obtuvo el promedio de las dos últimas; la presión sistólica se midió en la fase I de Korotkoff y la diastólica, en la fase $\mathrm{V}^{23}$

Estudios bioquímicos: los pacientes se presentaron en condiciones de ayunas de 10 a 12 horas. Se 
les tomó una muestra de sangre venosa del antebrazo en posición sentada sin anticoagulante, se separó el suero por centrifugación a 3500 rpm durante 5 minutos. Se determinaron los niveles de glicemia, triglicéridos y colesterol por métodos enzimáticos empleando reactivos estandarizados, y el colesterol HDL por precipitación con sulfato de dextran. ${ }^{24}$ El LDL se determinó con la fórmula de Friedewald. ${ }^{25}$

Se determinaron las frecuencia para cada categoría de riesgo coronario y del logro de las metas de colesterol LDL, se compararon las proporciones empleando la prueba $Z .^{23} \mathrm{Se}$ consideró significativo un $p<0,05$. El estudio fue aprobado por el Comité de Investigación de la Facultad de Medicina de la Universidad Nacional de Trujillo. Se realizó teniendo en cuenta las recomendaciones de Helsinki y el Código de Ética del CMP. Los participantes firmaron el acta de consentimiento informado.

\section{RESULTADOS}

Se estudió a una muestra de 552 personas, 276 varones y 276 mujeres. En la Tabla 1 se describen las características generales de las personas en el estudio. Se encontraron 128 personas con cintura hipertensiva $(23,2 \%), 55$ varones $(19,9 \%)$ y 73 mujeres $(26,4 \%)$.
La frecuencia de categoría de riesgo bajo es mayor en los pacientes sin cintura hipertensiva, en cambio las categorías de riesgo moderadamente alto y alto es mayor en los pacientes con cintura hipertensiva; el logro de la meta LDL colesterol es menor en los pacientes con cintura hipertensiva en las categorías de riesgo bajo, moderado y moderadamente alto (Tabla 2).

En los varones con cintura hipertensiva, la frecuencia de la categoría «riesgo bajo» disminuyó con el incremento de la edad, y las de moderadamente alto y alto aumentaron con la edad en forma significativa. En las mujeres se observó algo semejante, pero no fue significativo: en la categoría de riesgo bajo coronario, los valores fueron mayores en las mujeres y en la de riesgo alto, fueron menores que en los varones, pero sin diferencia significativa $(p=0,052)$. En el total de la población, la edad afectó la frecuencia de las categorías de riesgo, disminuyendo las de bajo riesgo y aumentando las de alto riesgo; $p=$ 0,0013 (Tabla 3).

En los varones con cintura hipertensiva, el logro de la meta de LDL fue mayor en la categoría de riesgo bajo y menor en la alta, así mismo, fue mayor en las personas más jóvenes $(p<0,00001)$. En las mujeres, el cumplimiento sigue la misma tendencia $(p=0,0810)$, pero el cumplimiento es

Tabla 1. Características de la población estudiada.

\begin{tabular}{lccc} 
& Varones & Mujeres & SE $(p)$ \\
$\Delta$ Edad & & & 92 \\
- 20-39 años & 92 & 92 \\
$-40-59$ años & 92 & 92 \\
$-60-79$ años & 92 & 276 & $<0,00001$ \\
Total & 276 & $24,68(3,74)$ & $<0,00001$ \\
$\Delta$ IMC $\left(\mathrm{kg} / \mathrm{m}^{2}\right)$ & $26,72(3,92)$ & $81,83(9,85)$ & 0,0036 \\
$\Delta$ Cintura $(\mathrm{cm})$ & $93,34(10,39)$ & $115,08(17,09)$ & $<0,00001$ \\
$\Delta$ PAS $(\mathrm{mmHg})$ & $119,75(14,71)$ & $71,86(10,52)$ & $<0,00001$ \\
$\Delta$ PAD $(\mathrm{mmHg})$ & $75,66(8,98)$ & $134,61(66,69)$ & 0,0444 \\
$\Delta$ Triglicéridos $(\mathrm{mg} / \mathrm{dl})$ & $170,66(109,26)$ & $215,84(45,83)$ & 0,0030 \\
$\Delta$ Colesterol total $(\mathrm{mg} / \mathrm{dl})$ & $208.26(42.48)$ & $138,46(42,60)$ & $<0,00001$ \\
$\Delta$ LDL $(\mathrm{mg} / \mathrm{dl})$ & $128.14(38.52)$ & $49,93(8,27)$ & 0,0097 \\
$\Delta$ HDL $(\mathrm{mg} / \mathrm{dl})$ & $46.25(8.19)$ & $92,66(25,28)$ & \\
\hline Glucosa basal $(\mathrm{mg} / \mathrm{dl})$ & $99,46(35,45)$ & & \\
\hline
\end{tabular}


Tabla 2. Categoría de riesgo coronario y logro de la meta de colesterol LDL en personas con y sin cintura hipertensiva Logro de la meta de colesterol LDL

\begin{tabular}{|c|c|c|c|c|c|c|c|c|}
\hline & \multicolumn{2}{|c|}{ Riesgo bajo } & \multicolumn{2}{|c|}{ Riesgo moderado } & \multicolumn{2}{|c|}{ Riesgo moderado alto } & \multicolumn{2}{|c|}{ Riesgo alto } \\
\hline & $\mathrm{CCH}$ & $\overline{\mathrm{SCH}}$ & $\mathrm{CCH}$ & $\mathrm{SCHr}$ & $\mathrm{CCH}$ & $\mathrm{SCH}$ & $\mathrm{CCH}$ & $\mathrm{SCH}$ \\
\hline \multicolumn{9}{|l|}{$\Delta$ Varones } \\
\hline - Frecuencia de categorías (\%) & 27,27 & 65,16 & 9,09 & 7,24 & 32,73 & 17,19 & 30,91 & 10,41 \\
\hline - Logro de la meta (\%) & 100 & 80,56 & 80 & 50 & 50 & 55,26 & 17,65 & 13,04 \\
\hline \multicolumn{9}{|l|}{$\Delta$ Mujeres } \\
\hline - Frecuencia de categorías (\%) & 39,73 & 84,65 & 27,40 & 8,91 & 17,81 & 1,49 & 15,07 & 4,95 \\
\hline - Logro de la meta (\%) & 44,83 & 78,36 & 20 & 61,11 & 38,46 & 33,33 & 9,09 & 10 \\
\hline \multicolumn{9}{|l|}{$\triangle$ Total } \\
\hline - Frecuencia de categorías (\%) & 34,38 & 74,47 & 15,63 & 8,04 & 24,22 & 9,69 & 21,88 & 7,80 \\
\hline - Logro de la meta $(\%)$ & 63,64 & 79,37 & 32 & 55,88 & 45,16 & 53,66 & 14,29 & 12,12 \\
\hline
\end{tabular}

$\mathrm{CCH}$ : con cintura hipertensiva. SCH: sin cintura hipertensiva

menor que en los varones en todas las categorías $(p=0,0083)$. En el total de la población se siguió la tendencia descrita $(p=0,0004)$ (Tabla 4).

En los varones sin cintura hipertensiva la categoría de riesgo bajo disminuyó con la edad y la moderadamente alto y alto aumentaron con la edad. En las mujeres se observó algo semejante, pero de menor intensidad; en ella, los valores de categoría bajo fueron mayores y de alto menores que los varones (Tabla 5). En las personas con

Tabla 3. Categorías de riesgo coronario en adultos de Trujillo con cintura hipertensiva.

\begin{tabular}{|c|c|c|c|c|c|c|c|c|c|c|}
\hline \multirow[b]{2}{*}{ Sexo y edad } & \multicolumn{2}{|c|}{ Riesgo bajo } & \multicolumn{2}{|c|}{ Riesgo moderado } & \multicolumn{2}{|c|}{ Riesgo moderado alto } & \multicolumn{2}{|c|}{ Riesgo alto } & \multicolumn{2}{|c|}{ Total } \\
\hline & $n$ & $\%$ & $\mathrm{n}$ & $\%$ & $\mathrm{n}$ & $\%$ & $\mathrm{n}$ & $\%$ & $\mathrm{n}$ & $\%$ \\
\hline \multicolumn{11}{|l|}{$\triangle$ Varones } \\
\hline$-20-39$ & 8 & 88,89 & 1 & 11,11 & 0 & & 0 & 0 & 9 & 100 \\
\hline$-40-59$ & 5 & 31,25 & 3 & 18,75 & 5 & 31,25 & 3 & 18,75 & 16 & 100 \\
\hline \multirow[t]{2}{*}{$-60-79$} & 2 & 6,67 & 1 & 3,33 & 13 & 43,33 & 14 & 46,67 & 30 & 100 \\
\hline & 15 & 27,27 & 5 & 9,09 & 18 & 32,73 & 17 & 30,91 & 55 & 100 \\
\hline \multicolumn{11}{|l|}{$\Delta$ Mujeres } \\
\hline$-20-39$ & 1 & 100,00 & 0 & & 0 & & 0 & & 1 & 100 \\
\hline$-40-59$ & 11 & 47,83 & 7 & 30,43 & 2 & 8,70 & 3 & 13,04 & 23 & 100 \\
\hline \multirow[t]{2}{*}{$-60-79$} & 17 & 34,69 & 13 & 26,53 & 11 & 22,45 & 8 & 16,33 & 49 & 100 \\
\hline & 29 & 39,73 & 20 & 27,40 & 13 & 17,81 & 11 & 15,07 & 73 & 100 \\
\hline \multicolumn{11}{|l|}{$\Delta$ Total } \\
\hline$-20-39$ & 9 & 100,00 & 1 & & 0 & & 0 & & 10 & 100 \\
\hline$-40-59$ & 16 & 41,03 & 10 & 20,51 & 7 & 17,95 & 6 & 15,38 & 39 & 100 \\
\hline$-60-79$ & 19 & 24,05 & 14 & 10,13 & 24 & 30,38 & 22 & 27,85 & 79 & 100 \\
\hline Total & 44 & 34,38 & 25 & 15,63 & 31 & 24,22 & 28 & 21,88 & 128 & 100 \\
\hline
\end{tabular}

Efecto de la edad en varones sobre la frecuencia de categorías de riesgo, $p<0,00001$; en mujeres, $p=0,6659$.

Frecuencia de bajo riesgo en varones vs mujeres: $p=0,2$; alto riesgo, $p=0,053$.

No hay diferencia significativa entre hombres y mujeres en la categoría de bajo riesgo coronario $(p=0,2)$. 
Tabla 4. Logro de la meta de colesterol LDL según sexo, grupo etario y categoría de riesgo en adultos de Trujillo con cintura hipertensiva

\begin{tabular}{|c|c|c|c|c|c|c|c|c|c|c|}
\hline \multirow[b]{3}{*}{ Sexo } & \multicolumn{2}{|c|}{$\begin{array}{l}\mathrm{LDL}<160 \mathrm{mg} / \mathrm{dl} \\
\text { (riesgo bajo) }\end{array}$} & \multicolumn{2}{|c|}{$\begin{array}{c}\mathrm{LDL}<130 \\
\text { (riesgo moderado) } \\
\end{array}$} & \multicolumn{2}{|c|}{$\begin{array}{c}\mathrm{LDL}<130 \mathrm{mg} / \mathrm{dl} \\
\text { (moderadamente alto) }\end{array}$} & \multicolumn{2}{|c|}{$\begin{array}{c}\mathrm{LDL}<100 \mathrm{mg} / \mathrm{dl} \\
\text { (riesgo alto) }\end{array}$} & \multicolumn{2}{|c|}{ Total } \\
\hline & Total & LM & Total & LM & Total & LM & Total & LM & Total & LM \\
\hline & $\mathrm{N}$ & $\%$ & $\mathrm{~N}$ & $\%$ & $\mathrm{~N}$ & $\%$ & $\mathrm{~N}$ & $\%$ & $\mathrm{~N}$ & $n$ \\
\hline
\end{tabular}

$\begin{array}{lrrrrrrrrrrrrrrr}\Delta \text { Varones } & & & & & & & & & & & & & & & \\ -20-39 & 8 & 8 & 100 & 1 & 1 & 100 & 0 & 0 & 0 & 0 & 0 & & 9 & 9 & 100 \\ -40-59 & 5 & 5 & 100 & 3 & 3 & 100 & 5 & 1 & 20 & 3 & 0 & 0 & 16 & 9 & 56,25 \\ -60-79 & 2 & 2 & 100 & 1 & 0 & 0 & 13 & 8 & 61,54 & 14 & 3 & 21,43 & 30 & 13 & 43,33 \\ \text { Total } & 15 & 15 & 100 & 5 & 4 & 80 & 18 & 9 & 50,00 & 17 & 3 & 17,65 & 55 & 31 & 56,36\end{array}$

$\triangle$ Mujeres

$\begin{array}{llllllllll}-20-39 & 1 & 1 & 100 & 0 & 0 & 0 & 1 & 1 & 100\end{array}$

$\begin{array}{llllllllllllllll}-40-59 & 11 & 4 & 36,36 & 7 & 0 & 0,00 & 2 & 0 & 0 & 3 & 0 & 0 & 23 & 4 & 17,39\end{array}$

$\begin{array}{llllllllllllllll}-60-79 & 17 & 8 & 47,06 & 13 & 4 & 30,77 & 11 & 5 & 45,45 & 8 & 1 & 12,5 & 49 & 18 & 36,73\end{array}$

$\begin{array}{llllllllllllllll}\text { Total } & 29 & 13 & 44,83 & 20 & 4 & 20,00 & 13 & 5 & 38,46 & 11 & 1 & 9,09 & 73 & 23 & 31,51\end{array}$

$\triangle$ Total

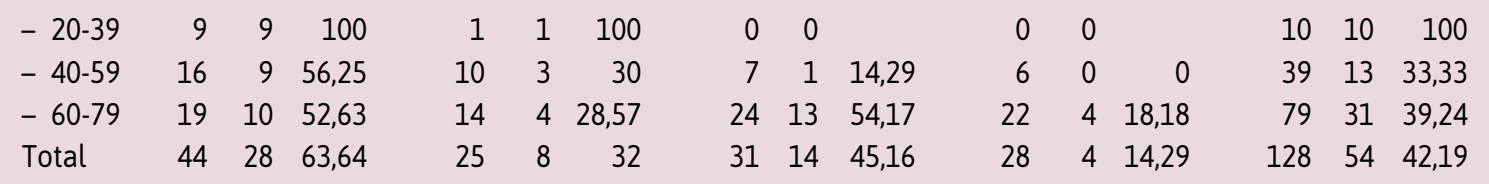

En varones, logro de la meta en riesgo bajo vs alto, $p<0,00001$; en mujeres, $p=0,0810$; total, $p=0,0004$.

$L M=$ Logro de la meta. Logro de la meta en varones vs. mujeres, $p=0,0083$.

Tabla 5. Categorías de riesgo coronario en adultos de Trujillo sin cintura hipertensiva

\begin{tabular}{|c|c|c|c|c|c|c|c|c|c|}
\hline & \multicolumn{2}{|c|}{ Riesgo bajo } & \multicolumn{2}{|c|}{ Riesgo moderado } & \multicolumn{2}{|c|}{ Riesgo moderado alto } & \multicolumn{2}{|c|}{ Riesgo alto } & \multirow{2}{*}{$\begin{array}{r}\text { Total } \\
\mathrm{N}\end{array}$} \\
\hline & $\mathrm{n}$ & $\%$ & $n$ & $\%$ & $n$ & $\%$ & $n$ & $\%$ & \\
\hline \multicolumn{10}{|l|}{$\triangle$ Varones } \\
\hline$-20-39$ & 81 & 97,59 & 1 & 1,20 & 0 & 0 & 1 & 1,20 & 83 \\
\hline$-40-59$ & 40 & 52,63 & 15 & 19,74 & 12 & 15,79 & 9 & 11,84 & 76 \\
\hline$-60-79$ & 23 & 37,10 & 0 & 0,00 & 26 & 41,94 & 13 & 20,97 & 62 \\
\hline 144 & 65,16 & 16 & 7,24 & 38 & 17,19 & 23 & 10,41 & 221 & \\
\hline \multicolumn{10}{|l|}{$\Delta$ Mujeres } \\
\hline$-20-39$ & 90 & 100,00 & 0 & 0,00 & 0 & 0,00 & 0 & 0,00 & 90 \\
\hline$-40-59$ & 56 & 81,16 & 10 & 14,49 & 0 & 0,00 & 3 & 4,35 & 69 \\
\hline$-60-79$ & 25 & 58,14 & 8 & 18,60 & 3 & 6,98 & 7 & 16,28 & 43 \\
\hline 171 & 84,65 & 18 & 8,91 & 3 & 1,49 & 10 & 4,95 & 202 & \\
\hline \multicolumn{10}{|l|}{$\triangle$ Total } \\
\hline$-20-39$ & 171 & 98,84 & 1 & 0,58 & 0 & 0,00 & 1 & 0,58 & 173 \\
\hline$-40-59$ & 96 & 66,21 & 25 & 17,24 & 12 & 8,28 & 12 & 8,28 & 145 \\
\hline \multirow[t]{2}{*}{$-60-79$} & 48 & 45,71 & 8 & 7,62 & 29 & 27,62 & 20 & 19,05 & 105 \\
\hline & 315 & 74,47 & 34 & 8,04 & 41 & 9,69 & 33 & 7,80 & 423 \\
\hline
\end{tabular}

Efecto de la edad en varones y mujeres, $p<0,00001$. Frecuencia de bajo riesgo varones vs mujeres, $p<0,0001$; alto riesgo, $p=0,0563$; la frecuencia de bajo riesgo vs alto riesgo en mujeres, $p=0,0016$. 
Tabla 6. Logro de la meta de colesterol LDL según sexo, grupo etario y categoría de riesgo en la población adulta de Trujillo sin cintura hipertensiva

\begin{tabular}{|c|c|c|c|c|c|c|c|c|c|c|c|c|c|c|c|}
\hline \multirow[b]{3}{*}{ Sexo } & \multicolumn{3}{|c|}{$\begin{array}{c}\mathrm{LDL}<160 \\
\text { (Riesgo bajo) }\end{array}$} & \multicolumn{3}{|c|}{$\begin{array}{c}\mathrm{LDL}<130 \\
\text { (Riesgo moderado) }\end{array}$} & \multicolumn{3}{|c|}{$\begin{array}{c}\mathrm{LDL}<130 \\
\text { (Moderado alto) }\end{array}$} & \multicolumn{3}{|c|}{$\begin{array}{c}\mathrm{LDL}<100 \\
\text { (Riesgo alto) }\end{array}$} & \multicolumn{3}{|c|}{ Total } \\
\hline & \multirow{2}{*}{$\begin{array}{c}\text { Total } \\
\mathrm{N}\end{array}$} & \multicolumn{2}{|c|}{ LM } & \multirow{2}{*}{$\begin{array}{c}\text { Total } \\
\mathrm{N}\end{array}$} & \multicolumn{2}{|r|}{ LM } & \multirow{2}{*}{$\begin{array}{l}\text { Total } \\
\mathrm{N}\end{array}$} & \multicolumn{2}{|r|}{ LM } & \multirow{2}{*}{$\begin{array}{c}\text { Total } \\
\mathrm{N}\end{array}$} & \multicolumn{2}{|r|}{ LM } & \multirow{2}{*}{$\begin{array}{c}\text { Total } \\
\mathrm{N}\end{array}$} & \multicolumn{2}{|c|}{ LM } \\
\hline & & $n$ & $\%$ & & $n$ & $\%$ & & $\overline{\mathrm{N}}$ & $\%$ & & $n$ & $\%$ & & $n$ & $\%$ \\
\hline \multicolumn{16}{|l|}{$\triangle$ Varones } \\
\hline$-20-39$ & 81 & 70 & 86,42 & 1 & 0 & 0 & 0 & 0 & 0 & 1 & 0 & 0 & 83 & 72 & 86,75 \\
\hline$-40-59$ & 40 & 30 & 75,00 & 15 & 8 & 53,33 & 12 & 5 & 41,67 & 9 & 1 & 11,11 & 76 & 66 & 86,84 \\
\hline$-60-79$ & 23 & 16 & 69,57 & 0 & 0 & 0 & 26 & 16 & 61,54 & 13 & 2 & 15,38 & 62 & 55 & 88,71 \\
\hline Total & 144 & 116 & 80,56 & 16 & 8 & 50 & 38 & 21 & 55,26 & 23 & 3 & 13,04 & 221 & 193 & 87,33 \\
\hline \multicolumn{16}{|l|}{ Mujeres } \\
\hline$-20-39$ & 90 & 84 & 93,33 & 0 & 0 & 0 & 0 & 0 & 0,00 & 0 & 0 & 0,00 & 90 & 84 & 93,33 \\
\hline$-40-59$ & 56 & 38 & 67,86 & 10 & 7 & 70 & 0 & 0 & 0,00 & 3 & 0 & 0,00 & 69 & 45 & 65,22 \\
\hline$-60-79$ & 25 & 12 & 48,00 & 8 & 4 & 50 & 3 & 1 & 33,3 & 7 & 1 & 14,29 & 43 & 18 & 41,86 \\
\hline Total & 171 & 134 & 78,36 & 18 & 11 & 61,11 & 3 & 1 & 33,3 & 10 & 1 & 10,00 & 202 & 147 & 72,77 \\
\hline \multicolumn{16}{|l|}{$\Delta$ Total } \\
\hline$-20-39$ & 171 & 154 & 90,06 & 1 & 0 & 0 & 0 & 0 & 0,00 & 1 & 0 & 0,00 & 173 & 156 & 90,17 \\
\hline$-40-59$ & 96 & 68 & 70,83 & 25 & 15 & 60 & 12 & 5 & 41,67 & 12 & 1 & 8,33 & 145 & 111 & 76,55 \\
\hline$-60-79$ & 48 & 28 & 58,33 & 8 & 4 & 50 & 29 & 17 & 58,62 & 20 & 3 & 15,00 & 105 & 73 & 69,52 \\
\hline Total & 315 & 250 & 79,37 & 34 & 19 & 55,88 & 41 & 22 & 53,66 & 33 & 4 & 12,12 & 423 & 340 & 80,38 \\
\hline
\end{tabular}

En varones y mujeres, logro de la meta en riesgo bajo vs alto, $p<0,00001$. Logro de la meta en varones vs mujeres, $p=0,0003$.

cintura hipertensiva la frecuencia de categorías de riesgo bajo fueron menores que las que no presentaban cintura hipertensiva, y las de categoría alta fueron mayores en ambos sexos y en el total de la población (Tabla 2).

En los varones sin cintura hipertensiva el logro de la meta de LDL fue mayor en la categoría de riesgo bajo y menor en la alta, ello no fue influenciado por la edad. En las mujeres el cumplimiento sigue la misma tendencia respecto a las categorías, pero fue mayor en las personas más jóvenes (Tabla 6). En las personas con cintura hipertensiva el cumplimiento de la meta de LDL fue menor en ambos sexos y en el total de la población respecto a los que no la presentaron.

\section{DISCUSIÓN}

Las características de la población del presente estudio son semejantes a los de estudios previos, $13,16,26$ donde los varones tienen mayor IMC, cintura, trigliceridemia, PAS, PAD y glicemia, mientras que las mujeres mayor colesterolemia, colesterol LDL y colesterol HDL. La frecuencia de la cintura hipertensiva encontrada es mayor en mujeres, similar a lo descrito por Nita, en Rumania, ${ }^{1}$ y Escobar, ${ }^{27}$ en nuestro medio. La frecuencia global $(23,14 \%)$ es menor a lo señalado por Nita (43,3\%), quien señala que la determinación de la cintura hipertensiva es un buen predictor para el síndrome metabólico, con una sensibilidad de $80,4 \%$ y una especificidad de $84 \%$; Escobar encuentra sensibilidad menor $(58,92 \%)$ y similar especificidad $(84,93 \%)$. De esta manera, la cintura hipertensiva es un examen preliminar valioso., ${ }^{1,27}$

En el presente trabajo se propuso evaluar el impacto de la presencia de cintura hipertensiva mediante la variación de la frecuencia de las categorías de riesgo coronario así como el cumplimiento de la meta colesterol LDL. Se encontró una mayor frecuencia de categorías de riesgo moderadamente alto y alto en los pacientes con cintura hipertensiva; al ser la cintura hipertensiva con sus dos componentes una definición breve del síndrome metabólico como lo relata Nita, ${ }^{1}$ se explica la tendencia encontrada, 
estando estos tres conceptos relacionados: cintura hipertensiva, síndrome metabólico y riesgo coronario; se esperaba que el incremento de uno genere el incremento de los otros. Estudios previos han demostrado que la presencia de síndrome metabólico genera una frecuencia mayor de categorías de riesgo coronario moderadamente alto y alto. ${ }^{26}$ El beneficio ulterior del concepto de cintura hipertensiva es su practicidad clínica, utilizando exclusivamente métodos que no constituyen ningún gasto para el paciente.

Está descrito que a mayor categoría de riesgo es menor el cumplimiento de la meta, tanto en la población general como con síndrome metabólico, ${ }^{12,26}$ en este trabajo este hecho se repite en toda la población, siendo más intenso en los pacientes con cintura hipertensiva en las categorías de riesgo bajo, moderado y moderadamente alto.

En la población general con cintura hipertensiva, se observa que el incremento de la edad aumenta la frecuencia de las categorías más altas y disminuye la frecuencia de las más bajas, este efecto ha sido descrito previamente en población general y con síndrome metabólico. ${ }^{12,26}$ El cumplimiento de la meta LDL colesterol en pacientes con cintura hipertensiva disminuyó con la edad y con el incremento de riesgo coronario en forma significativa en varones y fue inconstante en las mujeres, esto debido, probablemente, al tamaño de muestra del subgrupo etario de mujeres de 6079 años con categoría de riesgo moderadamente alto.

Los varones, que tienen la frecuencia mayor de categorías altas, tienen, mayor cumplimiento de la meta de LDL. Similar resultado encontraron en un estudio en diabéticos con cumplimiento de la meta con frecuencia similar, teniendo correlación con la adherencia al tratamiento. En otro estudio hubo menor cumplimiento de la meta, otras causas a estos hallazgos puede ser la falta de seguimiento. En el Lipid Treatment Assessment Project (L-TAP) solo hubo 38\% de cumplimiento en la meta de colesterol LDL, que varió según la categoría de riesgo: $68 \%$ en el bajo riesgo; $37 \%$ de alto riesgo y solo $18 \%$ de pacientes con enfermedad coronaria. En base a lo encontrado, se puede decidir la creación o implementación de programas educativos, incluso para pacientes con cintura hipertensiva y con categoría de riesgo bajo o moderado, a fin de evitar que se incremente su riesgo coronario, y mejorar por ende su adherencia al tratamiento para cumplir la meta LDL que repercutirá en un mejor estilo de vida. ${ }^{28-31}$

En forma complementaria al uso de la nueva guía de manejo de hipercolesterolemia, ${ }^{3}$ el empleo de las categorías de riesgo coronario y la cintura hipertensiva brinda al paciente y al personal de salud, el concepto dinámico de cambiar de una categoría de riesgo coronario alto a una menor mediante la modificación de los factores de riesgo coronario.

\section{CONCLUSIONES}

El impacto de la presencia de cintura hipertensiva es expresado al encontrar una mayor frecuencia de categoría de riesgo moderadamente alto y alto y un menor cumplimiento de la meta LDL en las categorías de riesgo bajo, moderado y moderadamente alto.

\section{RECOMENDACIONES}

Se sugiere difundir el empleo de la determinación de la cintura hipertensiva en despistaje de síndrome metabólico y de riesgo coronario (e incluirlo en los protocolos y guías de práctica clínica), por su bajo costo y alta aplicabilidad en todos los niveles de atención en nuestro país.

\section{AGRADECIMIENTOS}

Los autores agradecen a la señorita Mitzy Cerna y a la señora Teresa Delgado por el procesamiento de las muestras.

\section{REFERENCIAS BIBLIOGRÁFICAS}

I. Nita C, Hancu N, Rusu A, Bala C, Roman G. Hypertensive waist: first step of the screening for metabolic syndrome. Metab Syndr Relat Disord. 2009;7(2): 105-10.

2. Grundy SM, Cleeman JI, Merz CN, Brewer HB Jr, Clark LT, Hunninghake DB, et al; Coordinating Committee of the National Cholesterol Education Program. Implications of recent clinical trials for the National Cholesterol Education Program Adult Treatment Panel III Guidelines. J Am Coll Cardiol. 2004;44:720-32.

3. Stone NJ, Robinson JG, Lichtenstein AH, Bairey Merz CN, Blum $\mathrm{CB}$, Eckel RH, et al; American College of Cardiology/American Heart Association Task Force on Practice Guidelines. 2013 ACC/ AHA guideline on the treatment of blood cholesterol to reduce atherosclerotic cardiovascular risk in adults: a report of the American College of Cardiology/American Heart Association Task Force on Practice Guidelines. J Am Coll Cardiol. 2014;63(25 Pt B):2889-934. 
4. Expert Panel on Detection, Evaluation, and Treatment of High Blood Cholesterol in Adults. Executive Summary of The Report of The National Cholesterol Education Program (NCEP) Expert Panel on Detection, Evaluation, And Treatment of High Blood Cholesterol In Adults (Adult Treatment Panel III). JAMA. 200।;285(19):2486-97.

5. Romero T. ¿Cuál es el riesgo cardiovascular del adulto asintomático? Rev Chil Cardiol. 20 I2;3 I(2): I34-4I.

6. Alberti KG, Eckel RH, Grundy SM, Zimmet PZ, Cleeman Jl, Donato KA, et al. Harmonizing the metabolic syndrome: a joint interim statement of the International Diabetes Federation Task Force on Epidemiology and Prevention; National Heart, Lung, and Blood Institute; American Heart Association; World Heart Federation; International Atherosclerosis Society; and International Association for the Study of Obesity. Circulation. 2009;120(16):1640-5.

7. US Department of Health and Human Services, National Institute of Health, National High Blood Pressure Education Program. The Seventh Report of the Joint National Committee on Prevention, Detection and Treatment of High Blood Pressure. Bethesda (MD): National Heart, Lung, and Blood Institute (US); 2004.

8. Mancia G, Fagard R, Narkiewicz K, Redón J, Zanchetti A, Böhm $M$, et al. ESH/ESC Guidelines for the management of arterial hypertension: the Task Force for the management of arterial hypertension of the European Society of Hypertension (ESH) and of the European Society of Cardiology (ESC). J Hypertens. 20I3;3I(7): I28I-357.

9. ALLHAT Officers and Coordinators for theALLHAT Collaborative Research Group; The Antihypertensive and Lipid-Lowering Treatment to Prevent Heart Attack Trial. Major outcomes in moderately hypercholesterolemic, hypertensive patients randomized to pravastatin vs usual care:The Antihypertensive and Lipid-Lowering Treatment to Prevent Heart Attack Trial (ALLHATLLT). JAMA. 2002;288(23):2998-3007.

10. Sever PS, Dahlöf B, Poulter NR, Wedel H, Beevers G, Caulfield M, et al. Prevention of coronary and stroke events with atorvastatin in hypertensive patients who have average or lower-than-average cholesterol concentrations, in the Anglo-Scandinavian Cardiac Outcomes Trial--Lipid Lowering Arm (ASCOT-LLA): a multicentre randomised controlled trial. Drugs. 2004;64 Suppl 2:43-60.

II. Cannon CP, Braunwald E, McCabe CH, Rader DJ, Rouleau JL, Belder R, et al. Pravastatin or Atorvastatin Evaluation and Infection Therapy-Thrombolysis in Myocardial Infarction 22 Investigators. Intensive versus moderate lipid lowering with statins after acute coronary syndromes. N Engl ] Med. 2004;350(I5): 1495-504

12. Grundy SM, Cleeman Jl, Merz CN, Brewer HB Jr, Clark LT, Hunninghake DB, et al.; National Heart, Lung, and Blood Institute; American College of Cardiology Foundation; American Heart Association. Implications of recent clinical trials for the National Cholesterol Education Program Adult Treatment Panel III guidelines. Circulation. 2004; I 10(2):227-39.

13. Galarreta C, Donet MJ, Huamán SJ. Síndrome metabólico en la población adulta de Trujillo de acuerdo a diferentes definiciones Acta Med Per. 2009:26(4):217-225.

14. Huamán S], Castillo MK, Corrales PD, Reyes BM. Categorías de riesgo coronario y logro de la meta de LDL colesterol según edad y género en la población adulta de Trujillo, La Libertad, Perú 2007. Acta Med Per. 2008;25(2):68-73.

15. Exebio CC. Estadística aplicada a la investigación científica en ciencias de la salud. Trujillo, Perú: Cristóbal Exebio; 200I. p. 2I-35.

16. Linares-Reyes E, Castillo-Minaya K, Ríos-Mino M, HuamánSaavedra J. Estudio de correlación entre los diagnósticos de cintura hipertrigliceridémica y síndrome metabólico en adultos de Trujillo, Perú. Rev Peru Med Exp Salud Publica. 20I4;3I (2):254-
60.

17. Huamán SJ. Hiperlipidemia en una población laboral de Trujillo. Rev Med Per. 1997;69(357):44-8.

18. Huamán SJ. Dislipidemia en mujeres de 30 a 59 años. Rev Med Tru. 1998;2(2):27-35

19. Huamán SJ, CalderónAMC, Mestanza RPA,Tacanga LL. Distribución de la concentración sérica de lípidos en una población trujillana. Hampi Runa 2003;3(6):103-8.

20. Huamán SJ, Illatopa V, Calderón AMC, Huamán D RM. Factores de riesgo coronario en mujeres pre y postmenopáusicas. Hampi Runa 2004;4(9):45-54.

21. Huamán SJ. Prevalencia del síndrome metabólico en población adulta de Trujillo, según edad, género y algunos factores asociados. Acta Med Orreguiana Hampi Runa. 2007;7(I):4-I0.

22. American Diabetes Association. Executive summary: standards of medical care in diabetes--201I. Diabetes Care. 201 I;34 Suppl I:S4-10.

23. Chobanian AV, Bakris GL, Black HR, Cushman WC, Green LA, Izzo JL Jr, et al. The Seventh Report of the Joint National Committee on Prevention, Detection, Evaluation, and Treatment of High Blood Pressure: the JNC 7 report. JAMA. 2003;289(19):2560-72.

24. Warnick GR, Benderson J, Albers JJ. Dextran sulfate-Mg2+ precipitation procedure for quantitation of high-densitylipoprotein cholesterol. Clin Chem. 1982;28(6): 1379-88.

25. Friedewald WT, Levy RI, Fredrickson DS. Estimation of the concentration of low-density lipoprotein cholesterol in plasma, without use of the preparative ultracentrifuge. Clin Chem. 1972; 18(6):499-502.

26. Huamán J, Álvarez M, Ríos A. Factores y categorías de riesgo coronario y logro de la meta de LDL-colesterol según edad y género en pacientes con y sin síndrome metabólico en Trujillo. Rev Med Hered. 2012;23(3): 172-82.

27. Escobar P. Frecuencia de cintura hipertensiva y su concordancia con el síndrome metabólico en adultos de Trujillo. Tesis para optar el grado de bachiller en Medicina. Trujillo, Perú: Facultad de Medicina de la Universidad Nacional de Trujillo; 2013.

28. Parris ES, Lawrence DB, Mohn LA, Long LB. Adherence to statin therapy and LDL cholesterol goal attainment by patients with diabetes and dyslipidemia. Diabetes Care. 2005;28(3):595-9.

29. Straka RJ, Taheri R, Cooper SL, Tan AW, Smith AC. Assessment of hypercholesterolemia control in a managed care organization. Pharmacotherapy. 2001;2I(7):8I8-27.

30. Pearson TA, Laurora I, Chu H, Kafonek S. The lipid treatment assessment project (L-TAP): a multicenter survey to evaluate the percentages of dyslipidemic patients receiving lipid-lowering therapy and achieving low-density lipoprotein cholesterol goals. Arch Intern Med. 2000;160(4):459-67.

31. Krueger KP, Felkey BG, Berger BA. Improving adherence and persistence: a review and assessment of interventions and description of steps toward a national adherence initiative. J Am Pharm Assoc. (2003). 2003;43(6):668-78; quiz 678-9.

Danie

\section{Correspondencia}

Edgardo Linares-Reyes

dedalo83_8@hotmail.com

\section{Conflictos de interés}

El autor declara no tener conflictos de interés durante el planteamiento, ejecución de la investigación y la elaboración del artículo para su publicación.

Fecha de recepción: 01 de diciembre de 2014

Fecha de aceptación: 25 de octubre de 2015 